\title{
Flat-band localization in the Anderson-Falicov-Kimball model
}

\author{
A. M. C. Souza ${ }^{1}$ and H. J. Herrmann ${ }^{2,3}$ \\ ${ }^{1}$ Departamento de Fisica, Universidade Federal de Sergipe, 49100-000 Sao Cristovao-SE, Brazil \\ ${ }^{2}$ Departamento de Física, Universidade Federal do Ceará, 60451-970 Fortaleza, CE, Brazil \\ ${ }^{3}$ Computational Physics, IfB, ETH Hönggerberg, HIF E 12, CH-8093 Zürich, Switzerland
}

(Received 20 October 2008; revised manuscript received 5 February 2009; published 6 April 2009)

\begin{abstract}
The insulator-metal-insulator transition caused by a flatband is analyzed within dynamical mean-field theory using the Anderson-Falicov-Kimball model. We observe quantitative disagreement between the present approach and previous results. The presence of interactions enhances delocalization.
\end{abstract}

DOI: 10.1103/PhysRevB.79.153104

\section{INTRODUCTION}

Recent studies have shown a new disorder-induced metalinsulator transition (MIT) of one-electron states, called the "inverse Anderson transition." 1,2 The existence of this transition becomes already visible for noninteracting particles when the system has highly degenerated localized states forming a flatband. In a flatband localized states may melt into extended states due to disorder. For weak disorder, the localization is not a consequence of the strength of disorder but of the flatband. Increasing the degree of disorder localization-delocalization and delocalization-localization transitions appear.

Different aspects must appear when we also consider interactions between particles of the system. The present work addresses this issue. The investigation of this problem is particularly rich. The interaction causes a Mott-Hubbard MIT (Ref. 3) that competes with the Anderson transition. ${ }^{4}$

The inverse Anderson transition has been studied using various numerical techniques, among them, level statistics, ${ }^{5}$ $f(\alpha)$ characteristics of wave functions ${ }^{6}$ and the participation radius $^{7}$ framework. However, these powerful tools are not adequate for interacting systems in which matrices of high order must be exactly diagonalized restricting the study to extremely small systems.

The dynamical mean-field theory (DMFT) (Ref. 8) is a good tool to investigate the Mott-Hubbard-Anderson MIT in lattice electrons with local interactions and disorder. The Anderson transition has, for example, been explored on the Bethe lattice considering the Hubbard ${ }^{9}$ and Falikov-Kimball models. ${ }^{10}$ The metal and the insulator phases are detected by analyzing directly the local density of states (LDOS). The averaged LDOS can vanish in the band center at a critical disorder strength for a wide variety of averages. ${ }^{11,12}$ In particular, the arithmetic mean of this random one-particle quantity is noncritical at the Anderson transition and hence cannot help to detect the localization transition. By contrast, the geometric mean gives a better estimate of the averaged value of the LDOS, ${ }^{9,13}$ as it vanishes at a critical disorder strength and hence provides an explicit criterion for the Anderson localization. ${ }^{8,14,15}$ We have adopted the Hölder mean and analyzed how the averaged LDOS depends on the Hölder parameter.

In this paper, we investigate the disorder-induced insulator-metal transition in a flatband of the FalikovKimball model using the DMFT. A flatband is due to highly degenerated states. These states can be described by a com-
PACS number(s): 71.10.Fd, 71.27.+a, 71.30.+h

plete set of localized wave functions on a periodic lattice. The existence of a localized eigenstate generates a set of eigenstates with the same energy at equivalent positions of the periodic lattice. One finds a flatband within a specific subset of the parameter space describing the Hamiltonian in which all of the dispersion relations become flat. In the Falikov-Kimball model, a flatband can be obtained for the noninteracting mobile species on a diamond lattice with fourfold-degenerated orbitals. ${ }^{1}$ We present the ground-state phase diagram for different values of a parameter measuring the disorder strength and the dependence of the MIT transition on the Coulomb repulsion between fermions. Recently, we have applied the DMFT to this model. First, we showed that not only the geometric mean can offer a good approximation for the averaged LDOS providing an explicit criterion for Anderson localization. We found that the averaged LDOS can vanish in the band center at a critical disorder strength for a wide variety of generalized Hölder mean. ${ }^{11}$ Second, we have analyzed how the presence of the nextnearest-neighbor hopping influences the phase diagram of the ground state of this model, and, third, we have studied the main effects of the long-range correlated disorder. ${ }^{12}$

This paper is organized as follows. In Sec. II we introduce the Anderson-Falikov-Kimball model. The DMFT approach is described in Sec. III. In Sec. IV we present the results concerning the phase diagram. Finally in Sec. V we conclude.

\section{MODEL}

The Anderson-Falicov-Kimball model ${ }^{13}$ is a tight-binding model having two species of fermionic particles, mobile and immobile, which interact with each other when both are on the same lattice site. We introduce a local random potential for the mobile particles, giving rise to a competition between interaction and disorder. This model has been applied to mixed-valence compounds of rare earth, transition-metal oxides, binary alloys and metal ammonia solutions. ${ }^{16}$ Its Hamiltonian is

$$
H=\sum_{i} \epsilon_{i} c_{i}^{+} c_{i}-t \sum_{(i j)} c_{i}^{+} c_{j}+U \sum_{i} f_{i}^{+} f_{i} c_{i}^{+} c_{i},
$$

where $c_{i}^{+}\left(c_{i}\right)$ and $f_{i}^{+}\left(f_{i}\right)$ are, respectively, the creation (annihilation) operators for the mobile and immobile fermions at lattice site $i . \epsilon_{i}$ is a random potential describing the local disorder, $t$ is the electron transfer integral connecting nearest- 
neighbor sites, and $U$ is the Coulomb repulsion when mobile and immobile particles occupy the same site. We consider that the occupation of immobile particles is site independent having a probability $p=1 / 2$. The number of mobile particles on site $i$ is given by $n_{i}=c_{i}^{+} c_{i}$. A chemical-potential $\mu$ is introduced for the mobile subsystem to fix the system in the half-filled band $\left(\overline{n_{i}}=1 / 2\right)$. Here, the energy will be given in units of the hopping element $t$ (i.e., $t=1$ ).

The condition of finding a flatband is to find a set of the parameter space describing the Hamiltonian in which all of the dispersion relations become flat. For example, flatband has been found for tight-binding electrons on the hexagonal lattice in which each site has three orbitals. ${ }^{17}$ Also, singleelectron on the Hubbard model has a highly degenerated ground state on the Kagome lattice. ${ }^{18}$ In the Falikov-Kimball model, two degenerate bands can be obtained from a particular set of hopping, similarly to the method used in Ref. 17. A typical example is a diamond lattice with fourfolddegenerated orbitals. ${ }^{1}$ However, here the density of states come in as an initial condition of the DMFT, and thus we do not worry about the lattice that generate the flatband.

\section{DYNAMICAL MEAN-FIELD EQUATIONS}

The DMFT is calculated from the Hilbert transform,

$$
G(E)=\int \frac{d \omega N_{0}(\omega)}{\eta(E)-\omega+1 / G(E)},
$$

where $N_{0}(\epsilon)$ is the noninteracting density of states, $G(E)$ the translationally invariant Green's function, and $\eta(E)$ a hybridization function describing the coupling of a selected lattice site with the rest of the system. ${ }^{15}$

The band form is inserted in $N_{0}(\epsilon)$, in order that for the flatband the noninteracting density of states is

$$
N_{0}(E)=\frac{1}{2}\left[\delta\left(E-E_{0}\right)+\delta\left(E+E_{0}\right)\right],
$$

where the highly degenerated energies are $\pm E_{0}$. The DMFT allows us to choose arbitrarily $E_{0}$; however, its value depends directly on the lattice and on the set of parameters of the Hamiltonian for which the dispersion relations become flat. The two degenerate bands are obtained for the noninteracting mobile species in the absence of $f$ particles. The hopping $t$ is finite. It and the $E_{0}$ are obtained through the method used in Ref. 17.

The relation between $G(E)$ and $\eta(E)$ is obtained in a straightforward way from Eqs. (2) and (3),

$$
\eta(E)=\sqrt{\frac{1}{4 G(E)^{2}}+E_{0}^{2}}-\frac{1}{2 G(E)} .
$$

The LDOS is given by ${ }^{13}$

$$
P\left(E, \epsilon_{i}\right)=-\frac{1}{\pi} \operatorname{Im} G\left(E, \epsilon_{i}\right),
$$

where $G\left(E, \epsilon_{i}\right)$ is the local $\epsilon_{i}$-dependent Green's function. For the Anderson-Falicov-Kimball model we obtain that ${ }^{11}$

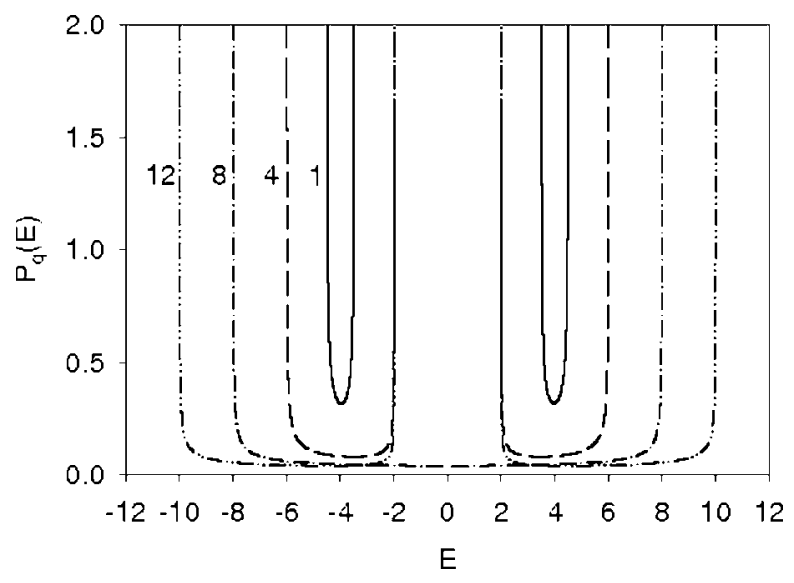

FIG. 1. Density of states for disorder strength $\Delta=0$ and $U=1,4$, 8 , and 12 .

$$
P\left(E, \epsilon_{i}\right)=-\frac{s}{\pi} \frac{\alpha_{i}^{2}+s^{2}+(U / 2)^{2}}{\left[\alpha_{i}^{2}+s^{2}+(U / 2)^{2}\right]^{2}-U^{2} \alpha_{i}^{2}},
$$

where $\alpha_{i}=E-\epsilon_{i}-r$ and $r$ and $s$ are, respectively, the real and imaginary parts of $\eta(E)$.

We consider that $\epsilon_{i}$ is an independent random variable characterized by a probability function $p\left(\epsilon_{i}\right)=\Phi(\Delta / 2$ $\left.-\left|\epsilon_{i}\right|\right) / \Delta$, with $\Phi$ being the step function. The parameter $\Delta$ is a measure for the disorder strength. The self-consistent DMFT equations are closed inserting

$$
G(E)=\int d \omega^{\prime} \frac{P_{q}\left(\omega^{\prime}\right)}{E-\omega^{\prime}},
$$

where

$$
P_{q}(E)=\left\{\sum_{i}\left[P\left(E, \epsilon_{i}\right)\right]^{q}\right\}^{1 / q} .
$$

The parameter $q$ defines the $q$-Hölder average. The arithmetic and geometric mean are found, respectively, using $q$ $=1$ and $q \rightarrow 0$.

The DMFT algorithm can be summarized as: (i) we choose an initial $P_{q}(E)$; (ii) from Eq. (7) we determine $G(E)$; (iii) from Eq. (4) we obtain $\eta(E)$; (iv) from Eq. (6) we find $P\left(E, \epsilon_{i}\right)$; and finally, (v) from Eq. (8) we have $P_{q}(E)$. This algorithm is iterated until we find the stable $P_{q}(E)$ function.

\section{RESULTS}

First, we investigate the case without disorder. In this limit, where $\Delta=0$, we find, independently of $q$, that the analytical expression for the density of states of the flatband is

$$
P_{q}(E)=\frac{E}{\pi \sqrt{\left[E^{2}-\left(E_{0}+U / 2\right)^{2}\right]\left[\left(E_{0}-U / 2\right)^{2}-E^{2}\right]}},
$$

if $\left|\left(E_{0}-U / 2\right)\right|<|E|<\left|\left(E_{0}+U / 2\right)\right|$ and $P_{q}(E)=0$, otherwise. Typical results are shown in Fig. 1. In order to compare our results with Refs. 1 and 2, we have set the highly degenerated state at $E_{0}=4$. Below $U=8$ we have two bands of bandwidth $U$. For $U>8$ we also have two bands, but now the bandwidth is constant and equal to 8 . The band gap is always 


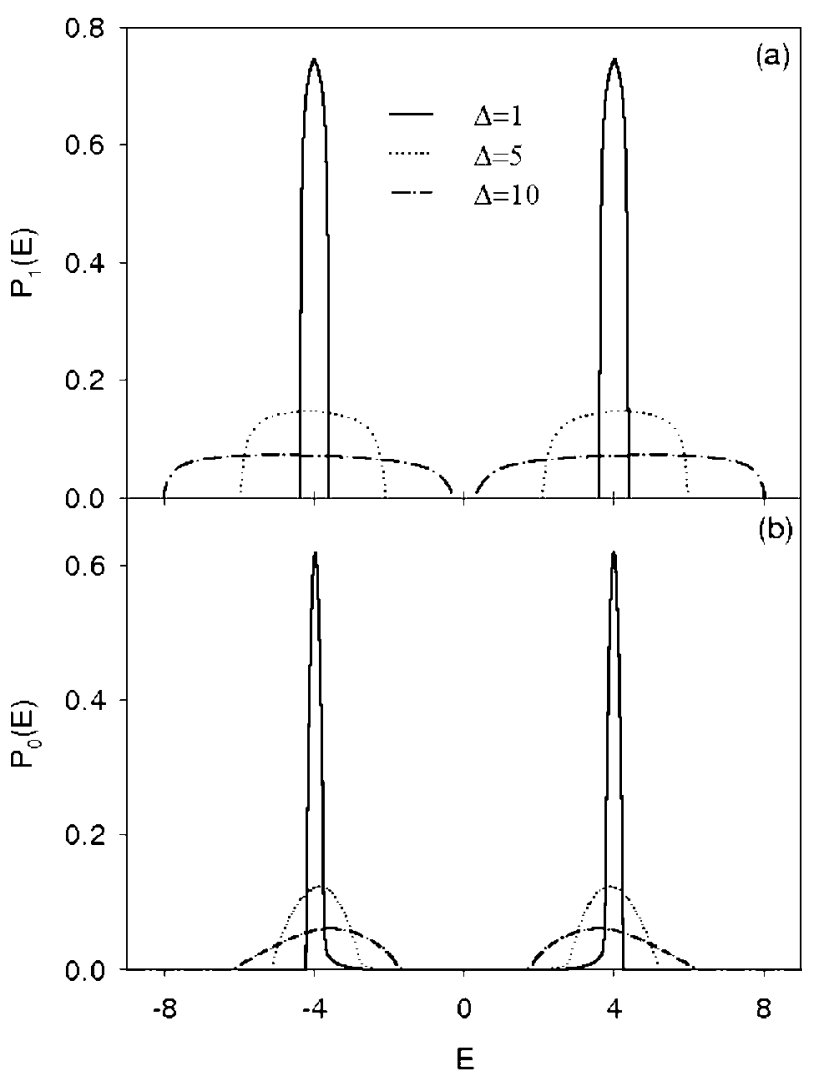

FIG. 2. Averaged local density of states at $U=0$ and disorder strength $\Delta=1,5$, and 10 for (a) $q=1$ and (b) $q=0$.

$|8-U|$. This band gap for $U \gg 1$ exhibits the Mott insulator relationship between the band gap and the repulsive Coulomb potential (band gap $\sim U$ ). Note that for $U=8$ the system presents only one band of bandwidth 8 , and only for this case $P_{q}(0) \neq 0$.

Next, let us explore the case $\Delta \neq 0$. Here, the results are obtained numerically. We considered as initial $P_{q}(E)$ a uniform distribution with bandwidth greater than the Lifshitz band edge. Then we determined $G(E)$ in order to obtain $\eta(E)$ and finally the new values of $P_{q}(E)$. This procedure is repeated until we find the stable configuration.

Figure 2 shows the energy dependence of the averaged LDOS for $U=0$ and typical values of $\Delta$. Note that the inclusion of the disorder (i.e., $\Delta \neq 0$ ) suppresses the highly degenerated localized states. We obtain the Anderson localization for a fixed $q$ varying the disorder strength $\Delta$ for each value of $U$, and then determining the values of $\Delta$ when $P_{q}(E)=0$. For the highly degenerated energy state, the arithmetic mean $(q$ $=1$ ) of the LDOS does not vanish at a finite critical disorder strength. Hence, we consider it to be noncritical at the Anderson transition. Using the geometric average $(q=0)$, the LDOS vanishes at the highly degenerated energy state for a finite value of $\Delta$. The detection of the Anderson localization is obtained using $q=0$.

Figure 3 presents the phase diagram of the ground state for the Anderson-Falikov-Kimball model as a function of energy for $U=0$ using the geometrical average. The results of Ref. 1 are shown in Fig. 3(a). Our results are presented in Fig. 3(b). As we use an iterative process, our $P_{q}(E)$ not al-

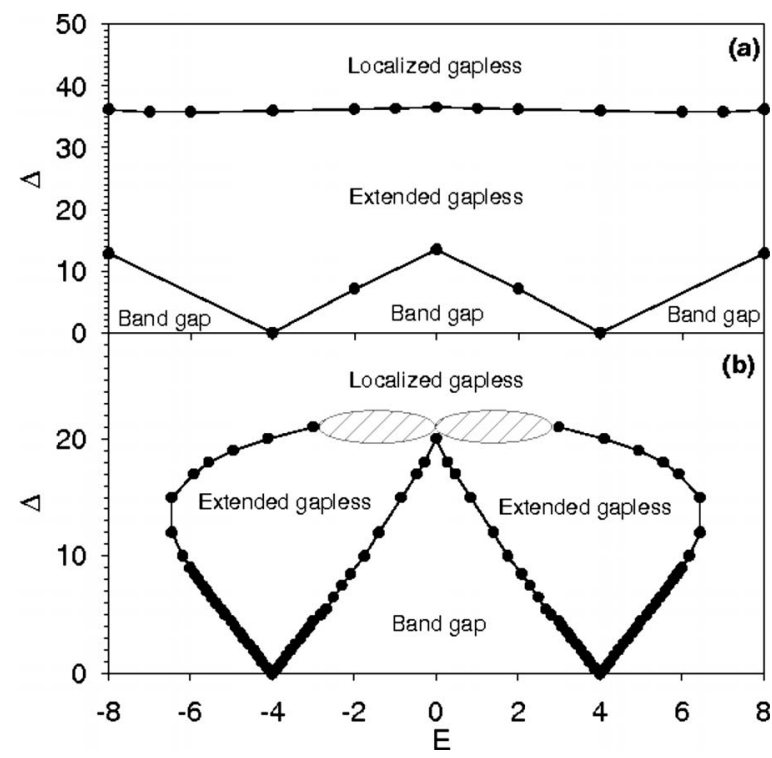

FIG. 3. Ground-state phase diagram as a function of energy for $U=0$. Case (a) was taken from Ref. 1. Case (b) was determined from the numerical solution of the DMFT equations for $q=0$. The dashed region is the energy area in which we cannot estimate the transition points accurately. Lines are guides to the eyes.

ways converges to a stable value for large $\Delta$. Within the dashed area of Fig. 3 we cannot estimate the transition points accurately. We can observe three phases: extended gapless, localized gapless, and band gap. ${ }^{13}$

The DMFT gives different results from the ones obtained in Refs. 1 and 2. The DMFT approach reduces the extended phase, increasing the critical disorder $\Delta_{c}$ for the localizationdelocalization transition and decreasing the one for the delocalization-localization transition. The considerable quantitative disagreement between both approaches requires new investigations to better understand these differences.

We now consider the influence of the Coulomb repulsion $U$ on the results. Figure 4 shows the averaged LDOS for $\Delta$ $=0.1,0.5,1.0$, and 5.0 at $U=1$. The results for $U=4$ are exhibited in Fig. 5, for $\Delta=1,2,5$, and 10. As already ob-

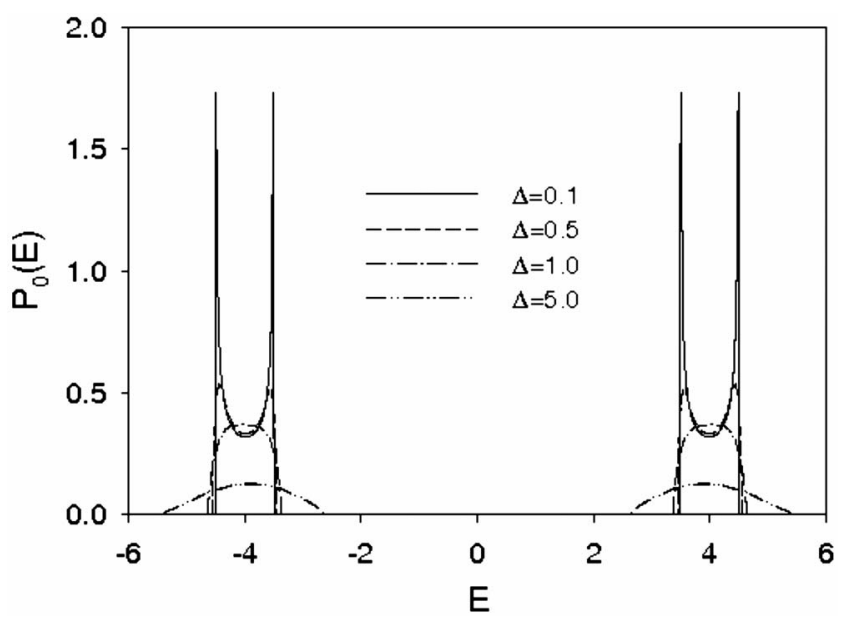

FIG. 4. Averaged local density of states at $U=1$ and disorder strength $\Delta=0.1,0.5,1.0$, and 5.0 for $q=0$. 


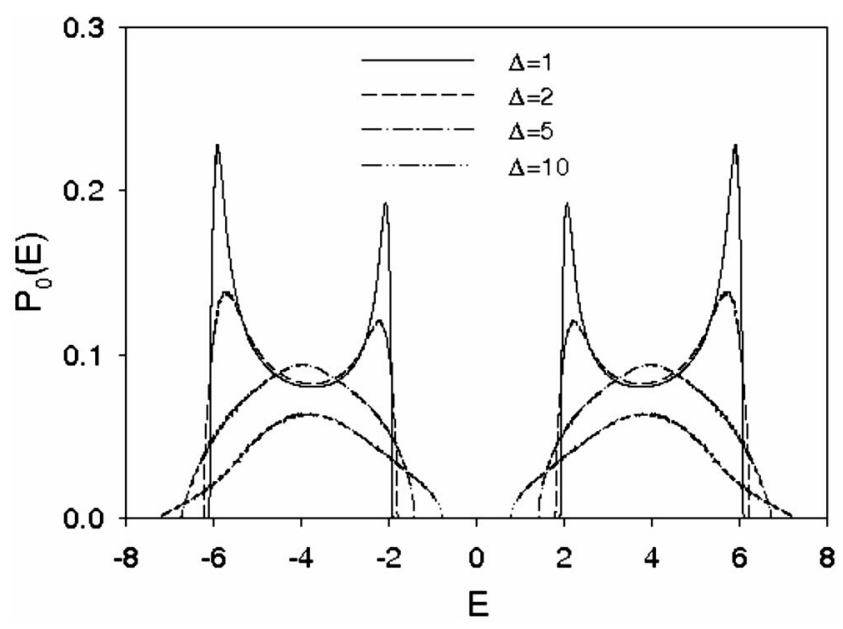

FIG. 5. Averaged local density of states at $U=4$ and disorder strength $\Delta=1,2,5$, and 10 for $q=0$.

served for $U=0$, we find two symmetric bands. However, for small $\Delta$, the LDOS at the end bands, corresponding to the smallest and largest energies, are higher than those at the band centers. The band centers correspond to the highly degenerated states for $U=0$. Large $\Delta$ destroy the influence of the flatband and favor higher LDOS values at band centers and smaller ones at the edge bands. When $\Delta$ is large enough, the LDOS vanish and the extended phase disappears. For fixed $\Delta$, the bandwidth grows with increasing $U$.

Finally, in Fig. 6 we present a complete ground-state phase diagram for three different values of $U$, namely, $U$ $=0,1$, and 4 . With $U$ increases the extended phase, showing that the interaction may enhance delocalization.

\section{CONCLUSIONS}

In this Brief Report, we studied the solutions of the Anderson-Falicov-Kimball model involving a highly degenerated localized states forming a flatband. We have shown that the present disorder-induced insulator-metal transition of one-electron states, called "inverse Anderson transition" can be obtained within dynamical mean-field theory. However, we observed a considerable quantitative disagreement be-

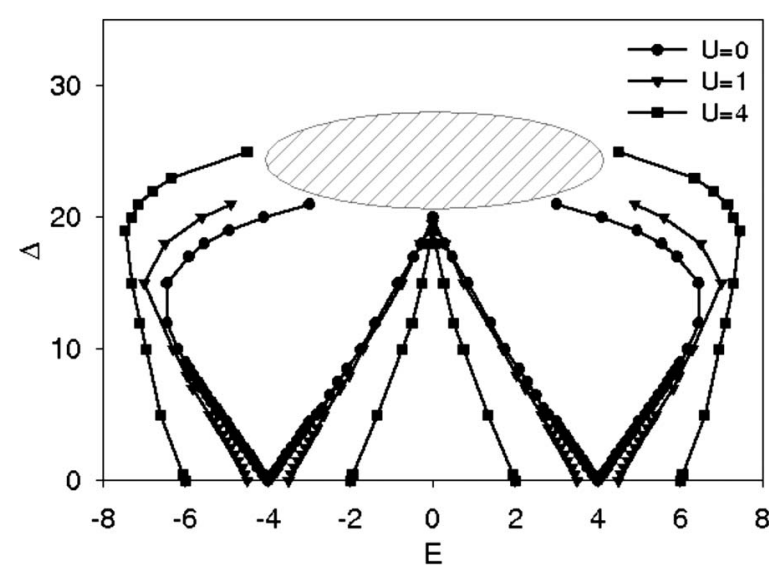

FIG. 6. Ground-state phase diagram as function of energy for $U=0,1$, and 4 obtained from the numerical solution of the DMFT equations. The dashed region is the energy area in which we cannot estimate the transition points accurately. Lines are guides to the eyes.

tween the present approach and the previous results. ${ }^{1,2}$

The DMFT reduces the extended phase, increasing the critical disorder $\Delta_{c}$ for the localization-delocalization transition and decreasing $\Delta_{c}$ for the delocalization-localization transition. Our analysis predicts a minimum of about $50 \%$ decrease in $\Delta_{c}$ with respect to the results of Refs. 1 and 2 for the delocalization-localization transition. For $|E|<6.5$, the localization-delocalization transition line shifting by a mostly about $50 \%$ to the two different approaches. For $|E|$ $>6.5$, whereas in our case the extended gapless region disappears, the results of Refs. 1 and 2 show this phase.

We also studied the dependence of the MIT transition on the interaction between particles of the model and showed that increasing the interaction parameter reduces the extended phase for $U=0$. As the doping of an impurity in the flatband has the same characteristics of the impurity states in the quantum Hall system, ${ }^{2}$ it would be interesting to do similar calculations for such a system.

\section{ACKNOWLEDGMENT}

Financial support of Conselho Nacional de Pesquisas Cientificas $(\mathrm{CNPq})$ is acknowledged.
${ }^{1}$ M. Goda, S. Nishino, and H. Matsuda, Phys. Rev. Lett. 96, 126401 (2006).

${ }^{2}$ S. Nishino, H. Matsuda, and M. Goda, J. Phys. Soc. Jpn. 76, 024709 (2007).

${ }^{3}$ N. F. Mott, Proc. Phys. Soc., London, Sect. A 62, 416 (1949).

${ }^{4}$ P. W. Anderson, Phys. Rev. 109, 1492 (1958).

${ }^{5}$ E. Hofstetter and M. Schreiber, Phys. Rev. B 48, 16979 (1993).

${ }^{6}$ A. Chhabra and R. V. Jensen, Phys. Rev. Lett. 62, 1327 (1989).

${ }^{7}$ M. Schreiber, Phys. Rev. B 31, 6146 (1985).

${ }^{8}$ V. Dobrosavljevic and G. Kotliar, Phys. Rev. Lett. 78, 3943 (1997).

${ }^{9}$ K. Byczuk, W. Hofstetter, and D. Vollhardt, Phys. Rev. Lett. 94, 056404 (2005).

${ }^{10}$ J. K. Freericks and V. Zlatic, Rev. Mod. Phys. 75, 1333 (2003).

${ }^{11}$ A. M. C. Souza, D. O. Maionchi, and H. J. Herrmann, Phys. Rev.
B 76, 035111 (2007).

${ }^{12}$ D. O. Maionchi, A. M. C. Souza, H. J. Herrmann, and R. N. da Costa Filho, Phys. Rev. B 77, 245126 (2008).

${ }^{13}$ K. Byczuk, Phys. Rev. B 71, 205105 (2005).

${ }^{14}$ M. Romeo, V. Da Costa, and F. Bardou, Eur. Phys. J. B 32, 513 (2003).

${ }^{15}$ V. Dobrosavljevic, A. A. Pastor, and B. K. Nikolic, Europhys. Lett. 62, 76 (2003).

${ }^{16}$ K. Leung and F. S. Csajkall, J. Chem. Phys. 108, 9050 (1998).

${ }^{17}$ S. Nishino, M. Goda, and K. Kusakabe, J. Phys. Soc. Jpn. 72, 2015 (2003).

${ }^{18}$ A. Mielke, J. Phys. A 24, L73 (1991); 24, 3311 (1991); 25, 4335 (1992); H. Tasaki, Phys. Rev. Lett. 69, 1608 (1992); A. Mielke and H. Tasaki, Commun. Math. Phys. 158, 341 (1993). 